\title{
Los gestos y la enseñanza de palabras poco familiares: ¿Cuándo emplean las maestras información gestual?
}

\author{
Alejandra Beatriz Menti, Universidad Nacional de Córdoba y Consejo Nacional de Investigaciones \\ Cientificas y Técnicas (CONICET) \\ Florencia Alam, Universidad de Buenos Aire y Consejo Nacional de Investigaciones Cientificas y \\ Técnicas (CONICET) \\ Argentina
}

Articulo recibido 11 de marzo de 2013, aceptado 12 de noviembre de 2013, versión final 15 de diciembre de 2013

\begin{abstract}
El presente trabajo tiene por objeto analizar los gestos que las maestras emplean en el tratamiento de vocabulario poco familiar en clases de Ciencias Sociales de la escuela primaria. El corpus está compuesto por secuencias interaccionales (Goodwin \& Heritage, 1990; Schegloff, 1990) incluidas en 12 situaciones de enseñanza videofilmadas en $1^{\circ}, 3^{\circ}$ y $5^{\circ}$ grado de 4 escuelas, dos rurales y dos urbanas de la provincia de Córdoba, Argentina. En las secuencias las maestras empleaban términos que eran poco familiares para los niños. Se llevó a cabo un análisis cualitativo (Strauss \& Corbin, 1991) que permitió comparar distintas secuencias en las que las maestras explican, definen o reparan las mismas palabras. Los resultados mostraron que, a diferencia de lo señalado en otros trabajos el uso de gestos por parte de las maestras no se encuentra únicamente asociado al término poco familiar empleado sino que se produce en respuesta a acciones verbales y/o gestuales de los niños que muestran una posible falta de comprensión conceptual.
\end{abstract}

\section{Introducción}

El presente trabajo se propone analizar los gestos que las maestras de nivel primario utilizan en el tratamiento de vocabulario no familiar. Desde la perspectiva psicolingüística, sociopragmática (Tomasello, 2003) y experiencial (Nelson, 2007), que constituye el encuadre del presente trabajo, se asume que la adquisición del vocabulario tiene lugar en los intercambios conversacionales en los que el sostén social y el contexto situacional, no verbal y lingüístico permite que el niño infiera el significado de las palabras que desconoce (Beals, 1997; Snow, 1972; Weizman \& Snow, 2001).

La importancia que desde este encuadre se le otorga a los distintos tipos de información disponibles para el aprendizaje de nuevas palabras coincide con los trabajos que desde el análisis conversacional y la sociolingüística interaccional han señalado la necesidad de atender en la interacción no solo a las expresiones verbales de los participantes sino a la interrelación de los distintos campos semióticos disponibles (Goodwin, 2007; Gumperz, 1982; Mondada, 2001). De acuerdo con estos autores, el sentido de las interacciones no se completa con los significados de las palabras sino que se construye en la yuxtaposición de las expresiones lingüísticas con otros sistemas 
semióticos, tales como los gestos, la dirección de la mirada, la orientación corporal, expresiones faciales, los movimientos de todo el cuerpo, la postura, la variación en la entonación y la particular estructuración del contexto de actividad, así como los artefactos involucrados en la actividad.

Desde la psicología cognitiva, McNeill $(1992,2005)$ fue uno de los primeros investigadores en estudiar, de modo sistemático, los gestos y su rol en las interacciones. McNeill realiza un análisis detallado de los gestos de la mano y plantea que a través de este tipo de movimientos gestuales el pensamiento de los interlocutores se pone de manifiesto en las interacciones. Desde esta línea de investigación, los gestos son definidos, específicamente, como los movimientos de las manos y los brazos que son interpretados por los otros como parte de lo que la persona dice (Gaythwaite, 2005; McNeill, 1992; Roth, 2001). Es importante destacar que esta corriente de investigación se diferencia claramente de otras al sostener que las expresiones lingüísticas y los gestos constituyen modos alternativos para expresar un mismo significado aunque de maneras distintas. En este sentido, McNeill (1992), afirma que mientras el habla es lineal, segmentada y compuesta por unidades más pequeñas, el gesto, por oposición, es global y sintético. En efecto, un solo gesto puede expresar un conjunto de significados. Estas modalidades alternativas resultan importantes, sobre todo cuando se presentan lagunas en el lexicón del hablante. En este caso, los gestos expresan visualmente las características del concepto aludido y constituyen así una modalidad expresiva apropiada que cubre el vacio lingüístico (Lemke, 1999; McNeill, 1992; Roth, 2001).

Distintas investigaciones enmarcadas en los conceptos teóricos de la psicología cognitiva, se han focalizado en el papel que cumplen los gestos durante la enseñanza y el aprendizaje (GoldinMeadow, Kim \& Singer, 1999; Goodwin, 2007; Migdalek \& Rosemberg, 2012; Roth, 2001, Singer \& Goldin-Meadow, 2005; entre otros). Así, por ejemplo, Roth (2001) identificó dos posibles funciones generales de los gestos en el contexto escolar. Por un lado, el docente puede valerse de la información proporcionada por los gestos que realizan sus alumnos para evaluar si han comprendido o no el tópico de la clase. Mientras que, por otra parte, los gestos del docente pueden servir de apoyo o recurso adicional para que los alumnos comprendan el discurso del maestro. Esta afirmación se basa en el hecho de que los gestos, al transmitir información en formato viso-espacial de modo simultáneo y sincronizado con el discurso (McNeill, 1992) refuerzan el mensaje transmitido. En consonancia con este planteo, otras investigaciones señalaron que las instrucciones orales acompañadas de gestos promueven mejor el aprendizaje que las mismas instrucciones transmitidas con ausencia de gestos (Gaythwaite, 2005; Singer \& Goldin-Meadow, 2005). Asimismo, como señalaron Migdalek y Rosemberg (2012), los gestos pueden contribuir a focalizar 
la atención de los niños en los aspectos centrales de la consigna.

Los trabajos que han analizado la relación entre el uso de gestos y el aprendizaje de vocabulario no familiar han atendido, en su mayoría, al empleo de los gestos que realizan los estudiantes (Crowder \& Newman, 1993; Lemke, 1999; Roth, 2000). Así por ejemplo, Lemke (1999) realizó un análisis cualitativo de fragmentos de una clase universitaria. Los resultados de este análisis pusieron de manifiesto que los interlocutores apelaban a los gestos cuando no encontraban disponibles en su lexicón mental el término científico específico que necesitaban en ese momento. En este sentido, los gestos funcionaron como una alternativa.

En esta misma línea, Roth (2000) analizó los gestos que realizaban los alumnos en clases de física del colegio secundario. Los resultados de este estudio mostraron, por un lado, que cuando los alumnos no poseían el vocabulario científico apropiado se valían de gestos para explicar y describir los conceptos científicos. Sin embargo, a medida que incrementaban el dominio del discurso científico, éste adquiría una importancia cada vez mayor y los gestos ya no procedían al habla sino que comenzaban a producirse en simultáneo.

Los trabajos que analizaron el uso de gestos para enseñar vocabulario no familiar no son muy numerosos. Por un lado, se llevaron a cabo estudios con niños de preescolar en situaciones de alfabetización en el hogar (Rosemberg \& Stein, 2009) o en las situaciones de intercambio entre niños generadas en el marco de un programa de niños tutores en alfabetización (Alam, Stein \& Rosemberg, 2011). Los resultados de este último trabajo mostraron que los niños tutores acompañaban las explicaciones de las palabras poco familiares con información proveniente de distintos campos semióticos, entre ellos, gestos deícticos e icónicos.

Por otro lado, entre los estudios experimentales, se destaca el trabajo de Singer y GoldinMeadow (2005), en el que compararon distintas situaciones en las que se enseñaba el mismo concepto matemático a alumnos de $3^{\circ}$ y $4^{\circ}$ grado de la escuela primaria. El propósito de esta investigación fue determinar si los alumnos se apoyaban o no en la información gestual proporcionada por los docentes durante las clases de matemáticas. Los resultados de esta investigación mostraron que los alumnos más beneficiados fueron aquellos a los que se les enseñó el nuevo concepto mediante el empleo de gestos que transmitían una información distinta a la expresada en el discurso. En tal sentido, las autoras señalan que mediante esta estrategia de enseñanza se ofrece a los alumnos un camino alternativo para resolver el problema en cuestión y, en consecuencia, resultan muy beneficiosos para el aprendizaje de los alumnos.

Como se pone de manifiesto en los trabajos reseñados, no se han registrado estudios que 
analicen los gestos que utilizan las maestras durante el tratamiento de vocabulario no familiar en situaciones naturales de enseñanza a lo largo de la escuela primaria. El objetivo de este trabajo es precisamente analizar los gestos que emplean las docentes de escuelas primarias cuando enseñan palabras no familiares a los niños.

\section{Metodología}

\section{Corpus}

El corpus de este estudio está conformado por secuencias interaccionales incluidas en 12 situaciones de enseñanza que tuvieron lugar durante el desarrollo de la unidad temática Tipos de trabajos, correspondiente al área de Ciencias Sociales. Las situaciones de enseñanza fueron registradas en $1^{\circ}, 3^{\circ}$ y $5^{\circ}$ de cuatro escuelas primarias, dos urbanas y dos rurales, de la provincia de Córdoba, Argentina. En las secuencias interaccionales las maestras empleaban términos que eran poco familiares o muy poco familiares para los niños. Para la categorización de las palabras como poco familiares o muy poco familiares se seleccionaron del discurso de las maestras las 15 palabras más frecuentes que conformaban el texto académico (Green, Weade \& Graham, 1988) de la unidad temática Tipos de trabajos en cada uno de los cursos observados. A partir de una escala de valoración subjetiva, un grupo de 160 jueces conformados por padres y maestros de cada escuela40 por cada comunidad-, valoró si las palabras más frecuentes pronunciadas por las maestras eran muy familiares, poco familiares o muy poco familiares a los niños (Menti y Rosemberg, 2013).

\section{Obtención de información empírica}

Las situaciones de enseñanza fueron registradas por medio de videofilmaciones realizadas por el investigador presente en la situación. Las 12 maestras observadas recibieron únicamente la indicación de desarrollar la unidad temática del modo en el que lo harían si el investigador no estuviera presente.

\section{Transcripción de los datos}

Las videofilmaciones fueron transcritas para su análisis, realizando una desgrabación literal de las expresiones lingüísticas, como así también de la información del contexto situacional y de los comportamientos no verbales. Las transcripciones se realizaron de acuerdo al formato CHAT (Codes for the Human Analysis of Transcripts) según las normas estipuladas por el sistema CHILDES (Child Language Data Exchange System) desarrollado por MacWhinney y Snow (1985). 
Para la presentación de los ejemplos en el presente trabajo se utilizaron las convenciones descritas en el Anexo 1.

\section{Procedimientos de análisis de datos}

Se consideró para el análisis la secuencia interaccional (Goodwin \& Heritage, 1990; Schegloff, 1990) en la que se empleaban las palabras poco familiares. Empleando el programa CLAN se extrajo del corpus total de datos la información del entorno lingüístico y no lingüístico en el que se producían cada uno de los términos. Una vez identificadas las secuencias, se llevó a cabo un análisis cualitativo que combina el Método Comparativo Constante (Glaser \& Strauss, 1967; Strauss \& Corbin, 1991) con el empleo heurístico de conceptos del análisis de la conversación (Goodwin, 2007; Sacks, Schegloff \& Jefferson, 1974), de la sociolingüística interaccional (Gumperz, 1982) y de los estudios de los gestos (McNeill, 1992, 2005). Este análisis permitió comparar las secuencias en las que las maestras explican, definen o reparan el vocabulario no familiar.

\section{Resultados y discusión}

Los resultados mostraron que las maestras de ambos entornos -rurales y urbanos- y en los tres grados analizados $-1^{\circ}, 3^{\circ}$ y $5^{\circ}$ - realizan distintos tipos de gestos cuando explican, definen o reparan una palabra poco familiar a los niños. La comparación entre las secuencias en las que las docentes utilizan los términos seleccionados mostró que un mismo término podía estar acompañado por el uso de gestos en una secuencia y en otra no, tal como se muestra en los Ejemplos 1 y 2 en el que el término foco es proceso

Ejemplo 1: Secuencia interaccional de una clase de $1^{\circ}$ grado en una escuela rural.

$\{$ La maestra conversa con los niños sobre los distintos trabajos que se realizan en el campo. Les pregunta por los trabajos que realizan sus padres. Una de las niñas se acerca a la maestra y le cuenta algo sobre la siembra que no se escucha en la grabación, la maestra se dirige a toda la clase retomando lo que la niña dice\}

1. Maestra: \{se dirige a la clase\} para levantarla pero antes para que salga de debajo de la tierra ¿qué ha pasado? (.) ¿qué pasó antes?

2. Lautaro: la saca y lo que hace- lo que mi papá hace es cavarla

brazo como si

[hace un gesto con la mano y el

3. Maestra: a ver estuviera cavando]

4. Pablo: él trabaja trabaja él trabaja \{Gritando\}

5. Maestra: sh:: 


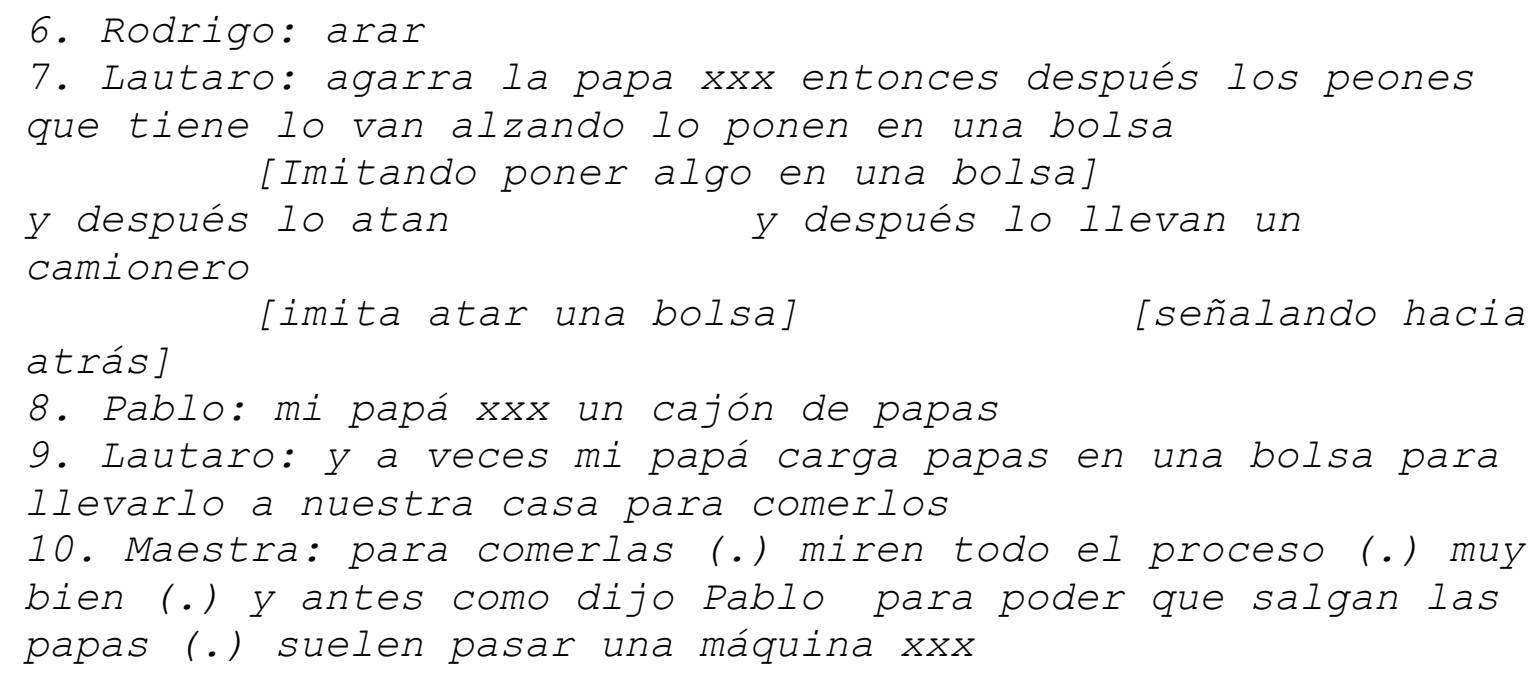

Como se muestra en el intercambio frente a la pregunta de la maestra por los trabajos que realizan los padres en el campo uno de los niños, Lautaro, explica el proceso de cosecha que realiza su padre (turno 2, 7 y 9): cava la tierra, agarra la papa, los peones la alzan y la ponen en una bolsa y la atan, y luego se lo lleva un camionero, y a veces el padre lleva papas a su casa para comer. Al relatar el proceso, el niño acompaña sus emisiones verbales con gestos icónicos (McNeill, 1992) que recuperan las acciones realizadas por los trabajadores del campo. Cabe señalar que en ningún momento el niño reemplaza las palabras por gestos, mostrando así que conoce y utiliza con frecuencia estos términos (Roth, 2000). Una vez que el niño termina de explicar (turno 9) la maestra señala verbalmente (turno 10, miren todo el proceso) que la explicación del niño responde a un proceso, palabra que si bien fue categorizada por los jueces poco familiar para los niños, refiere a un proceso que sí es familiar para ellos: la cosecha. Como se pone de manifiesto en el intercambio, la maestra no recurre a información gestual para acompañar el término. Esto contrasta con la secuencia que se presenta a continuación.

Ejemplo 2: Secuencia registrada en la misma clase que en el ejemplo 1 ( $1^{\circ}$ grado, escuela rural) $\{$ Después de conversar sobre los trabajos que se realizan en el campo la maestra propone comenzar a hablar de los trabajos que se realizan en la ciudad\}

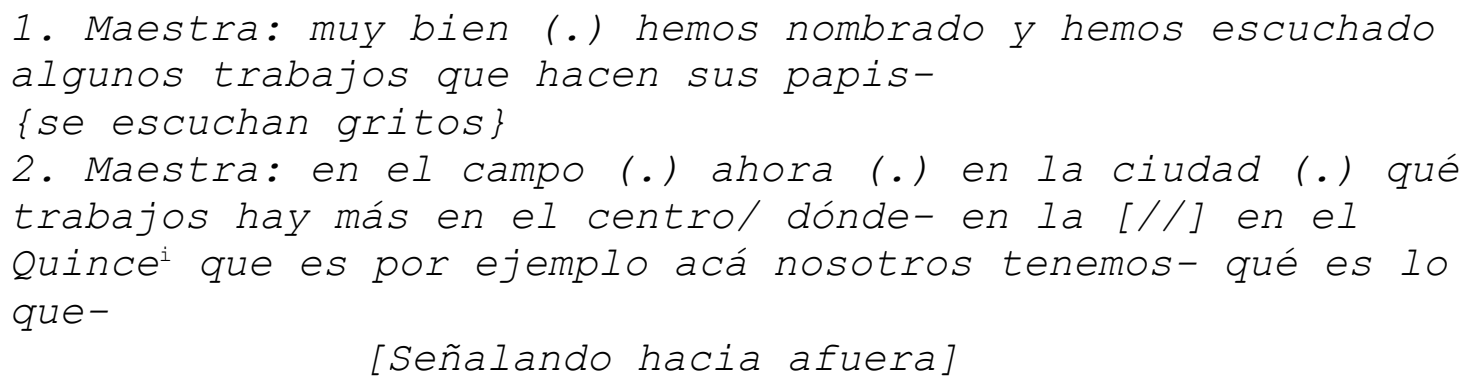




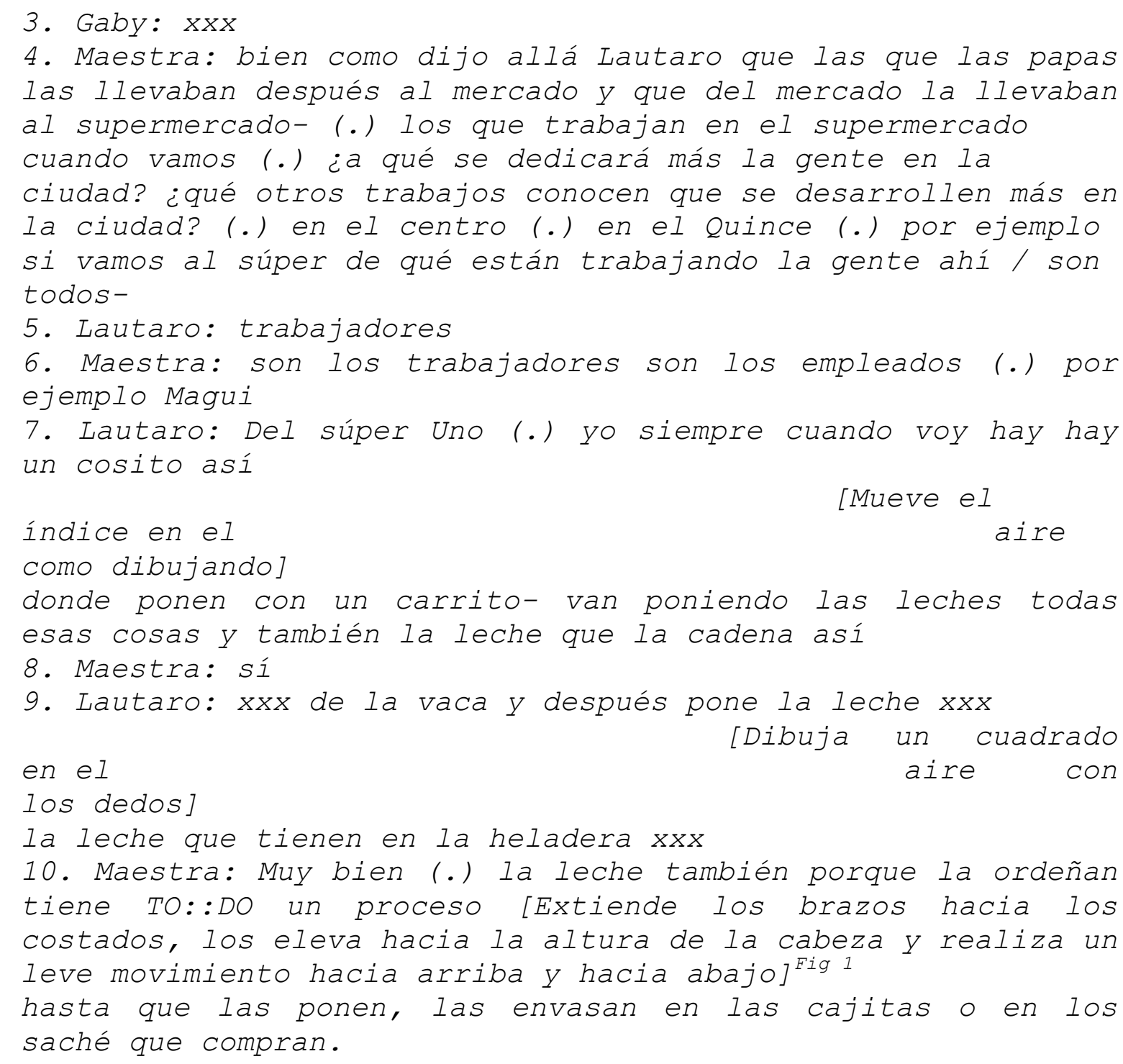

En el intercambio presentado, la maestra nuevamente introduce la palabra proceso que, en este caso, alude a las distintas fases implicadas en la comercialización de la leche, desde que sale del tambo hasta que llega a las góndolas del supermercado. El proceso completo en sí está resumido en la emisión (turno 10) de la maestra ya que ella sólo menciona la primera fase del proceso -la leche también porque la ordeñan-, y la última, -hasta que las ponen, las envasan en las cajitas o en los saché que compran-. A diferencia de la secuencia presentada en el Ejemplo 1, en el Ejemplo 2 la maestra yuxtapone al término abstracto proceso información gestual y prosódica. Al mismo tiempo que comienza a pronunciar la frase: tiene TO::DO un proceso, empieza a elevar el tono de la voz, poniendo mayor énfasis al pronunciar la palabra to::do. Cuando comienza a prolongar la primera vocal del cuantificador to::do la maestra extiende los brazos hacia la altura de la cabeza, realiza un tenue movimiento vertical -hacia arriba y hacia abajo- hasta finalizar la frase, tal como se observa 
en los siguientes fotogramas:

Figura 1: Gesto realizado por la maestra Maestra: tiene TO::DO un proceso

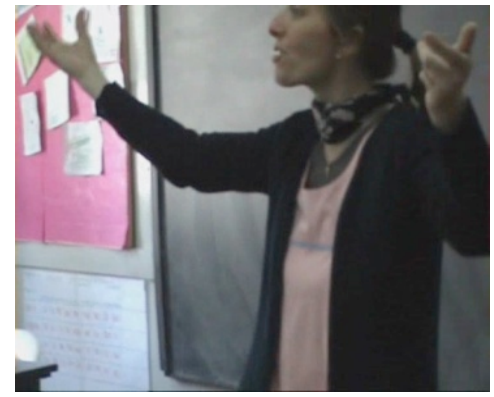

Figura 1.1

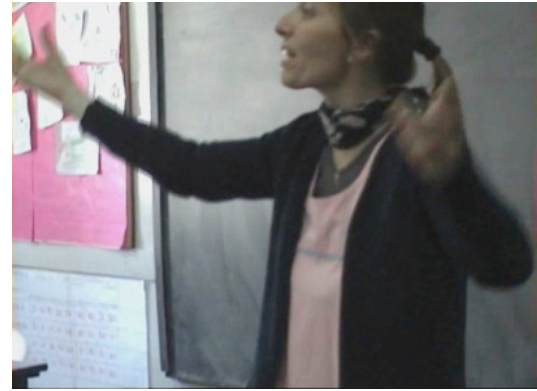

Figura 1.2

En este caso, la maestra realiza un gesto metafórico (McNeill, 1992) mediante el cual hace referencia a un aspecto del concepto abstracto, es decir, a la longitud del proceso, a la vez que señala los dos extremos de ese proceso que le permiten unir el campo y la ciudad. Es posible pensar que, a diferencia de lo que se observa en el intercambio anterior sobre la cosecha, el empleo del gesto en esta secuencia, puede responder a que la maestra asume el desconocimiento o un conocimiento sólo parcial por parte de los niños del proceso de comercialización en la ciudad.

$\mathrm{Si}$ analizamos los gestos y los términos que el niño emplea cuando se refiere al supermercado -turno 8, hay un cosito así [Mueve el índice en el aire como dibujando] (...) todas esas cosas y también la leche que la cadena asi- vemos que, a diferencia del proceso de cosecha donde el mismo niño emplea términos precisos que dan cuenta de un dominio del léxico -cavar, alzar, atar, bolsa, peones, camionero-, en la descripción del supermercado el niño utiliza gestos que reemplazan la palabra [Mueve el índice en el aire como dibujando]- y apoya su descripción en el uso de deícticos - así- y palabras poco precisas -cosito, esas cosas-.

Como señalaron varios trabajos, el uso de gestos en reemplazo de las palabras (Lemke, 1999; McNeil, 1992; Roth, 2000), así como el empleo de expresiones deícticas y palabras poco precisas (Menti \& Rosemberg, 2009; Rosemberg \& Silva, 2009) puede dar cuenta de lagunas léxicas. En este sentido, es posible pensar que tanto la falta de precisión léxica como los gestos empleados por el niño le permiten a la maestra considerar la falta de familiaridad de los niños con el proceso considerado, dando lugar así a focalizar la atención de los niños, a través de información verbal, prosódica y gestual, en el origen y el destino de dicho proceso.

La comparación entre los Ejemplos 1 y 2 permite pensar que el empleo de gestos por parte de la maestra responde a la secuencia conversacional en la que se insertan y no al término verbal 
que acompaña el gesto. Como sostienen diversos estudios (Goodwin \& Heritage, 1990; Schegloff, 1990; Unamuno, 2008) en una secuencia cada participante analiza las acciones de los otros para poder producir a su vez acciones que respondan a las realizadas previamente y que al mismo tiempo proyecten nuevas acciones. En efecto, el gesto que la maestra realiza no se explica simplemente en relación con el término proceso, ya que como muestra el Ejemplo 1 este término es también empleado sin un gesto que lo acompañe, sino por las intervenciones anteriores de los niños que permiten en un caso (Ejemplo 1) pensar que conocen y están habituados con el origen y el destino del proceso, y en el otro (Ejemplo 2) que dicho proceso es poco familiar. Si bien es posible que la maestra conozca la falta de familiaridad de los niños con el proceso de comercialización de la leche, conocimiento que puede tener por el trabajo diario con ellos, es en la interacción donde dicha falta se pone en evidencia a través del uso impreciso del vocabulario y del empleo de gestos en lugar de palabras.

El siguiente intercambio, que se presenta en el Ejemplo 3, fue registrado en $3^{\circ}$ grado de una escuela urbana. En este caso el término poco familiar empleado por la maestra es zona. La maestra inicia la secuencia recordándoles a los niños la actividad que debían hacer: averiguar qué fábricas hay en la zona en la que viven. Como se pone de manifiesto en el ejemplo la maestra no recurre a gestos cuando emplea el término foco.

Ejemplo 3: Intercambio registrado en $3^{\circ}$ grado de una escuela urbana

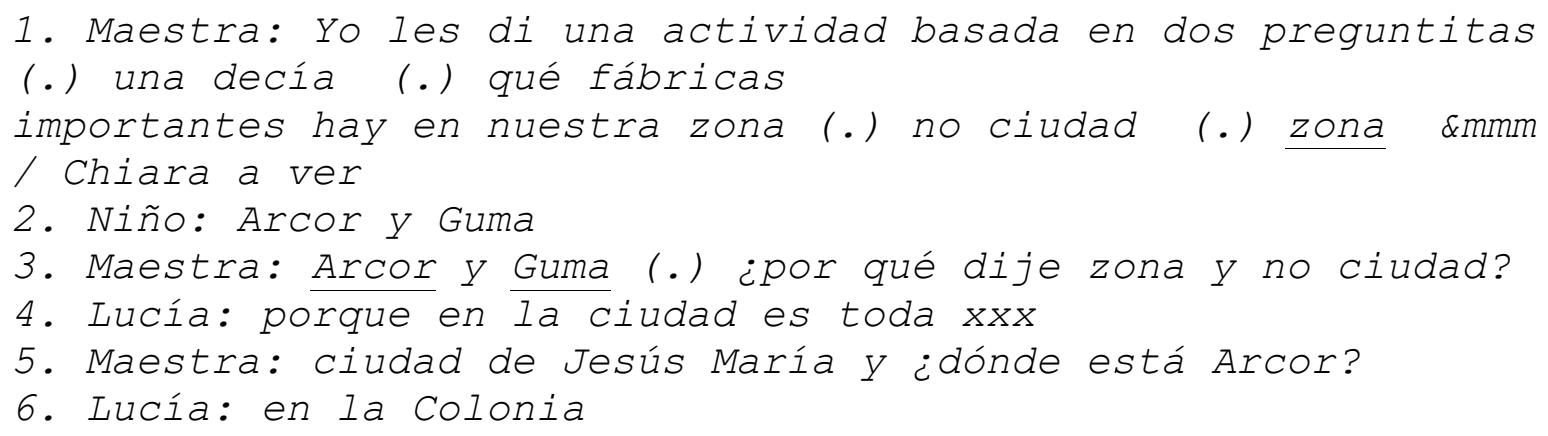

Como se muestra en los turnos 1 -en nuestra zona (.) no ciudad (.) zona-y 3 - ¿por qué dije zona y no ciudad? - la maestra parece reconocer que el término puede resultar problemático para los niños ya que existe la posibilidad de que lo confundan con la palabra ciudad. Sin embargo, luego de la respuesta de los niños en los turnos 2 -Arcor y Guma- y 4 -porque en la ciudad es toda xxx- la maestra parece dar por supuesto que los niños tienen clara la diferencia entre ambos conceptos. Asimismo, en el turno 5 la maestra diferencia la ciudad de Jesús María de la ciudad donde se 
encuentra la fábrica de Arcor, la Colonia. De este modo, deja implícito que ambas ciudades se encuentran en la misma zona. Así, para poder entender la diferencia entre zona y ciudad los niños deben inferir que los dos lugares nombrados -Jesús María y la Colonia- son dos ciudades distintas pero pertenecientes a la misma zona.

A continuación, los niños siguen nombrando más fábricas. Sin embargo, a partir de una de las respuestas, la maestra reconoce que algunos de los niños no han realizado la inferencia necesaria y, por tanto, no han comprendido completamente el término zona. En consecuencia, realiza una reparación que se presenta en el Ejemplo 4.

Ejemplo 4: Intercambio registrado durante la misma clase que el Ejemplo 3 ( $3^{\circ}$ grado, escuela urbana)

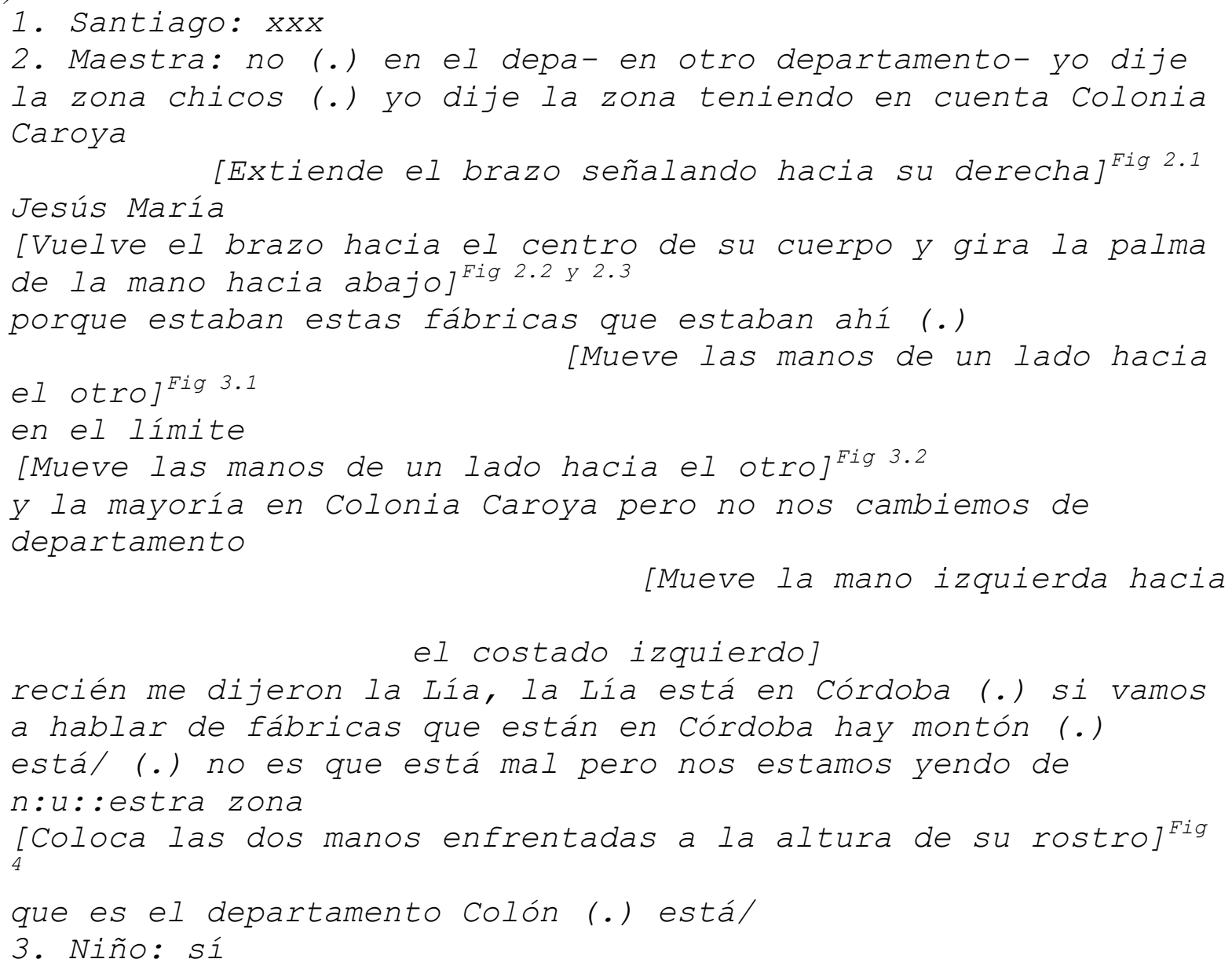


Figura 2: Gesto realizado por la maestra

Maestra: yo dije la zona teniendo en cuenta Colonia Caroya Jesús María

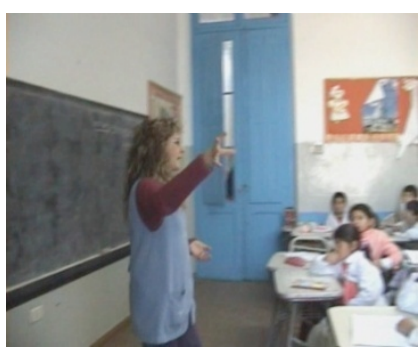

Figura 2.1

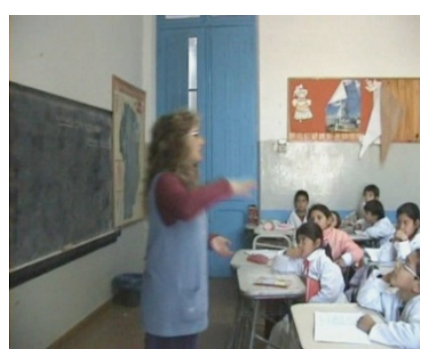

Figura 2.2

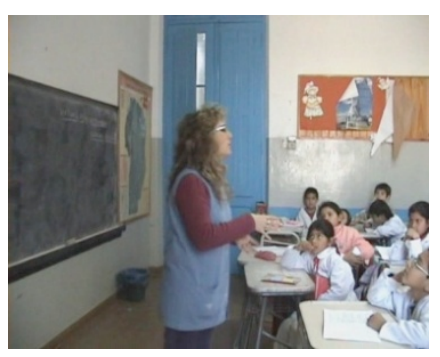

Figura 2.3

Como se muestra en la Figura 2, la maestra realiza gestos metafóricos mediante los cuales señala la ubicación de las dos ciudades mencionadas, Colonia Caroya y Jesús María. En el primer fotograma (Figura 2.1) la maestra aleja su brazo del cuerpo y lo extiende hacia un costado mientras menciona el nombre de la ciudad vecina, Colonia Caroya, ciudad alejada del contexto de situación. En el segundo fotograma (Figura 2.2), se presenta la trayectoria de la mano-brazo durante el momento de transición entre el primer gesto y el segundo. En el tercer fotograma (Figura 2.3), se puede observar cómo la maestra acerca y baja la mano-brazo hacia el centro de su cuerpo y gira la palma de la mano hacia abajo mientras menciona el nombre de la ciudad de Jesús María, ciudad donde está ubicada la escuela. Es importante destacar que ambos gestos transmiten información extra a la proporcionada por el discurso de la maestra, puesto que, en la explicación verbal la maestra no emplea deícticos lingüísticos para hacer referencia a la localización de las ciudades.

La maestra luego se refiere al espacio fronterizo entre ambas ciudades -estas fábricas que estaban ahí (.) [Mueve las manos de un lado hacia el otro] en el límite [Mueve las manos de un lado hacia el otro]-. Nuevamente, acompaña la información verbal con un gesto metafórico tal como se muestra en la Figura 3.

Figura 3: Gesto realizado por la maestra

Maestra: estas fábricas que estaban ahi (.) en el límite

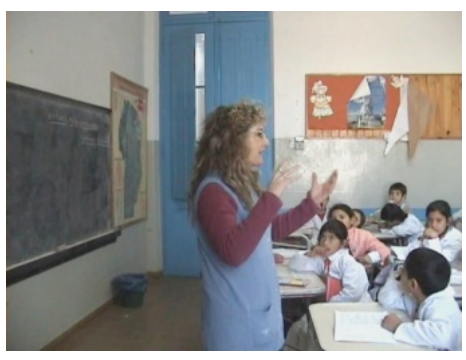

Figura 3.1

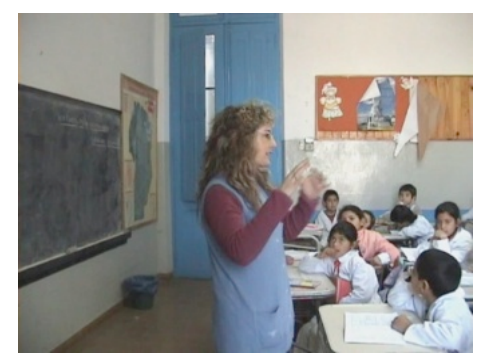

Figura 3.2

Finalmente, la maestra retoma el concepto nuestra zona. En este caso, realiza un 
prolongamiento vocálico del término nuestra, probablemente destinado a enfatizar que éste es el foco del tema que están realizando. Asimismo, yuxtapone a la emisión lingüística un gesto metafórico: acerca sus manos hacia la altura de su rostro y forma una esfera con ambas manos, tal como se muestra en la Figura 4.

Figura 4: Gesto realizado por la maestra

Maestra: no es que está mal pero nos estamos yendo de n:u::estra zona que es el departamento de Colón

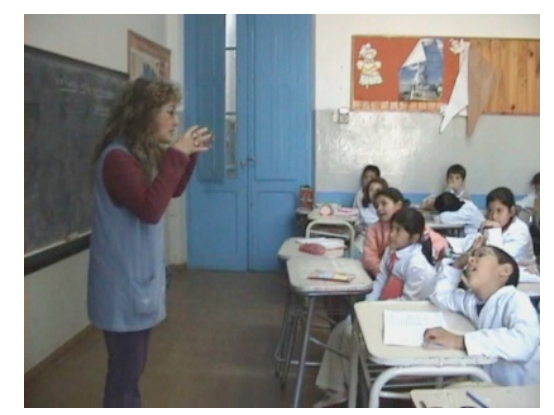

En todos los gestos realizados por la maestra durante su reparación se pone de manifiesto su intención de delimitar los espacios y de ponerlos en relación. Es posible entender este énfasis en la delimitación de los espacios en función de que éste es justamente el problema que presenta el término para los niños. Como sostiene Roth (2001) los gestos del docente pueden servir de apoyo para que los niños comprendan la información verbal, en tanto que al transmitir, de modo simultáneo con el discurso, información en formato viso-espacial (McNeill, 1992) refuerzan, y permiten aclarar, el mensaje transmitido.

Como se pone de manifiesto en el intercambio analizado la maestra percibe a partir de las respuestas de uno de los niños -turno 1- que hay un problema de comprensión en el término zona y realiza un movimiento de reparación. Diversos autores (Macbeth, 2004; Menti \& Rosemberg, 2011; Schegloff, 1992; Schegloff, Jefferson \& Sacks, 1977) han señalado que la reparación implica un problema en la comprensión mutua y un intento por parte de los interlocutores por restablecerla. En este sentido, la reparación da cuenta de la organización secuencial de la interacción. En efecto, el hablante realiza la reparación en función de la intervención de su interlocutor en la que reconoce el problema y que lo lleva, con el objetivo de mantener la comprensión mutua, a realizar su siguiente acción: reparar ese el problema.

En este caso, y al igual que en el análisis de los Ejemplos 1 y 2, se pone en evidencia que el empleo de los gestos responde a las acciones de los participantes en la secuencia. En este sentido, es 
posible pensar que la maestra no recurre al uso de gestos en el Ejemplo 3 cuando inicia la secuencia, aún cuando reconoce que el término puede ser complejo, porque en función de las primeras repuestas de los niños - Ejemplo 3, turno 2, Arcor y Guma; turno 4, porque en la ciudad es toda xxx; y turno 6, en la Colonia- considera que el término no resulta problemático. Sin embargo, al reconocer la falta de comprensión, a través de las acciones de sus interlocutores (Ejemplo 4), emplea gestos que permiten reparar el concepto, con el fin de que los niños puedan comprenderlo. El gesto, entonces, no solo se construye como respuesta a las intervenciones de sus interlocutores, sino también como proyección de nuevas acciones: la comprensión y el aprendizaje de términos abstractos. De esta manera, es posible entender el empleo de gestos por parte de la maestra a través del concepto de posición siguiente (Goodwin \& Heritage, 1990). De acuerdo con este concepto -que recupera y amplía la noción de par adyacente (Sacks, Schegloff \& Jefferson, 1974) -cada participante analiza las acciones de los otros para poder producir acciones que respondan a las realizadas previamente y que a la vez proyecten nuevas acciones.

\section{Conclusión}

El análisis de los gestos empleados por las maestras en el tratamiento de vocabulario poco familiar puso de manifiesto que las docentes emplean gestos para explicar, reparar o definir los términos no familiares a los niños. Sin embargo, la comparación entre secuencias en las que las docentes introducían un término poco familiar mostró que, a diferencia de lo señalado en trabajos anteriores que relacionan el uso de gestos con las palabras empleadas por el hablante (Singer \& GoldinMeadow, 2005), no es posible entender el uso de los gestos sólo en función del término verbal al que acompañan, sino en función de la secuencia interaccional. En efecto, el análisis de los intercambios mostró que las maestras empleaban gestos cuando los niños, a partir de sus acciones verbales y gestuales, mostraban una falta de comprensión conceptual. Considerar el empleo del gesto en relación únicamente con las acciones verbales que realiza el hablante no permite dar cuenta de la complejidad de las interacciones orales en las que todos los participantes, y no únicamente el hablante, analizan las acciones de sus interlocutores para poder producir acciones recíprocas y proyectar a su vez nuevas acciones (Goodwin y Heritage, 1990; Schegloff, 1990; Unamuno, 2008).

Las implicancias pedagógicas de este estudio resultan evidentes cuando se consideran en el marco de investigaciones previas que mostraron la importancia de las interacciones entre el adulto y el niño en el aprendizaje del vocabulario (Nelson, 1996, 2007; Snow, 1972; Tomasello, 1998, 2003; Weizman \& Snow, 2001). Como señalaron estos trabajos tanto la cantidad, la diversidad y la complejidad de las palabras que el adulto presenta al niño como el andamiaje que éste proporciona 
resultan relevantes para el aprendizaje de vocabulario. En este sentido, el presente estudio pone de manifiesto la necesidad de atender no solo a la información verbal que el adulto le puede proporcionar al niño cuando introduce o define un término poco familiar, sino también al empleo de gestos que le permitan al niño inferir y comprender el significado de las palabras que desconoce. Estos resultados pueden ser retomados en contextos de formación docente con el objeto de pensar estrategias que impliquen el empleo de distintos tipos de información en la enseñanza de vocabulario no familiar.

\section{Referencias bibliográficas}

Alam, F.; Stein, A. \& Rosemberg, C.R. (2011). "Te explico qué quiere decir", "te digo cómo se llama". Interacciones niño a niño en torno al vocabulario no familiar. Bellaterra Journal of Teaching \& Learning Language \& Literature, 4(4), 59-71.

Beals, D.E. (1997). Sources of support for learning words in conversation: evidence from mealtimes. Journal Child Language, 24(3), 673-694. doi:10.1017/S0305000997003267

Crowder, E. M. \& Newman, D. (1993). Telling what they know: The role of gesture and language in children's science explanations. Pragmatics \& Cognition, 1(2), 341-376. http://dx.doi.org/10.1075/pc.1.2.06cro

Gaythwaite, E.S. (2005). Didn't you see what I meant? Curriculum \& Teaching Dialogue, 7(1/2), 97-108.

Glaser, B.G. \& Strauss, A.L. (1967) The discovery of grounded theory. Chicago: Aldine Publishing Company.

Goldin-Meadow, S., Kim, S., \& Singer, M. (1999). What the teacher's hands tell the student's mind about math. Journal of Educational Psychology,91, 720-730. http://dx.doi.org/10.1037//00220663.91.4.720

Goodwin, C. (2007). Participation, stance and affect in the organization of activities. Discourse \& Society, 18(1), 53-73. http://dx.doi.org/10.1177/0957926507069457

Goodwin, C. \& Heritage, J. (1990). Conversation analysis. Annual review of anthropology, 19, 238307. http://dx.doi.org/10.1146/annurev.anthro.19.1.283

Green, J. Weade, R. \& Graham, K. (1988). Lesson construction and student participation: A sociolinguistic analysis. In J. Green \& J.O. Harke (Eds), Multiple perspective analysis of classroom discourse (pp. 11-47). Norwood, New Jersey: Ablex Publishing Corporation.

Gumperz, J. (1982). Discourse strategies. Cambridge: Cambridge University Press. http://dx.doi.org/10.1017/CBO9780511611834

Lemke, J. L. (1999). Typological and Topological Meaning in Diagnostic Discourse. Discourse Processes, 27(2), 173-185. http://dx.doi.org/10.1080/01638539909545057

Macbeth, D. (2004). The relevance of repair for classroom correction. Language in Society, 33, 703-736. http://dx.doi.org/10.1017/S0047404504045038

McNeill, D. (1992). Hand and mind: What gestures reveal about thought. Chicago: University of Chicago Press.

McNeill, D. (2005). Gesture and thought. Chicago: University of Chicago Press. http://dx.doi.org/10.7208/chicago/9780226514642.001.0001

MacWhinney, B. \& Snow, C. (1985). The child language data exchange system. Journal of Child Language, 12, 271- 296. http://dx.doi.org/10.1017/S0305000900006449

Menti, A. \& Rosemberg, C.R. (2009, Abril). La conversación en el aula: Oportunidades para el 
aprendizaje de vocabulario. En Carranza, I. (Comp), Actas del IV Coloquio de Investigadores en Estudios del Discurso y I Jornadas Internacionales de Discurso e Interdisciplina (pp. 9951002). Córdoba: Facultad de Lenguas.

Menti, A. \& Rosemberg, C.R. (2011). La enseñanza del vocabulario en las clases de ciencias: Análisis de los movimientos conversacionales de las docentes. Lenguaje, 39(1), 93-112.

Menti, A. \& Rosemberg, C.R. (2013). Oportunidades para el aprendizaje de vocabulario en las situaciones de enseñanza de ciencia sociales en la escuela primaria. Un estudio del vocabulario al que se hallan expuestos los niños. Interdisciplinaria - Revista de Psicología y Ciencias Afines, 30(2), 201-218.

Migdalek, M.J., \& Rosemberg, C.R. (2012). El uso de los gestos en el discurso docente durante la planificación del juego en el jardín de infantes. Bellaterra Journal of Teaching \& Learning Language \& Literature, 5(3), 25-43.

Mondada, L. (2001). Por una lingüística interaccional. Discurso y Sociedad, 3(3), 61-89.

Nelson, K. (2007). Young Minds in Social Worlds. Experience, Meaning and Memory. Cambridge: Harvard University Press.

Rosemberg, C.R. \& Silva, M.L. (2009). Teacher-Children Interaction and Concept Development in

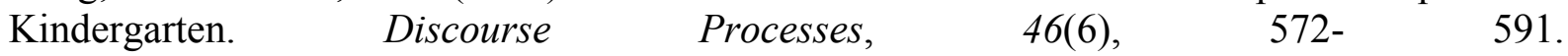
http://dx.doi.org/10.1080/01638530902959588

Rosemberg, C.R., \& Stein, A. (2009). Vocabulario y alfabetización temprana. Un estudio del entorno lingüístico en hogares de poblaciones urbano-marginadas. En M.C. Richaud \& J.E. Moreno (Eds), Investigación en Ciencias del Comportamiento (pp. 517-541). Buenos Aires: Ediciones CIIPME-CONICET.

Roth, W.M. (2000). From gesture to scientific language. Journal of Pragmatics, 32(11), 1683-1714. http://dx.doi.org/10.1016/S0378-2166(99)00115-0

Roth, W.M. (2001). Gestures: Their role in teaching and learning. Review of Educational Research, 71, 365-392. http://dx.doi.org/10.3102/00346543071003365

Sacks, H. Schegloff, E. A. y G. Jefferson (1974). A simplest systematic for the organization of turntaking for conversation. Language,50, 696-735. http://dx.doi.org/10.2307/412243

Schegloff, E.A. (1990). On the organization of sequences as a source of coherence in talk-in-interaction. In B. Dorval (Ed), Conversational Organization and its Development (pp. 51-77). Norwood, NJ: Ablex.

Schegloff, E.A. (1992). Repair after next turn: The last structurally provided defence on intersubjectivity in conversation. American Journal of Sociology 97, 1295-1345. http://dx.doi.org/10.1086/229903

Schegloff, E.A., Jefferson, G., \& Sacks, H.E. (1977). The preference for self-correction in the organization of repair in conversation. Language, 53(2), 361-382. http://dx.doi.org/10.2307/413107

Singer, M.A. \& Goldin-Meadow, S. (2005). Children learn when their teacher's gestures and speech differ. Psychological Science, 16, 85-89. http://dx.doi.org/10.1111/j.0956-7976.2005.00786.x

Snow, C.E. (1972). Mothers' speech to children learning language. Child Development, 43, 539565.

Strauss, A., \& Corbin, J. (1991). Basics of cualitative research. Grounded theory. Procedures and technics. Londres: Sage Publications.

Tomasello, M. (2003). Constructing a Language: A Usage-Based Theory of Language Acquisition. Massachusetts: Harvard University Press.

Unamuno, V. (2008). Multilingual switch in peer classroom interaction. Linguistics and Education, 19, 1-9. http://dx.doi.org/10.1016/j.linged.2008.01.002

Weizman, Z.O. \& Snow, C.E. (2001). Lexical input as related to children's vocabulary acquisition: 
Effects of sophisticated exposure and support for meaning. Developmental Psychology, 37(2), 265-279.

i

Así se le llama a las manzanas que conforman el centro de la ciudad de Colonia Caroya, ubicada a $50 \mathrm{~km}$ de la capital de Córdoba.

\section{Anexo 1: Pautas de transcripción}

: vocal prolongada

::: incremento en la prolongación de la vocal

$((\mathrm{XXX}))$ acción no verbal

[gesto] gesto realizado con la mano-brazo

$\{\mathrm{XX}\}$ comentarios del observador

$¿ X$ ? Entonación ascendente para interrogaciones

/ entonación ascendente

subrayado énfasis

MAYÚSCULAS mayor énfasis

(.) pausa breve

xxx emisión incomprensible

Alejandra Beatriz Menti es doctora en Ciencias del Lenguaje con Mención en Lingüística Aplicada por la Universidad Nacional de Córdoba, Argentina. Actualmente es becaria postdoctoral del Consejo Nacional de Investigaciones Científicas y Técnicas (CONICET- Argentina) y profesora asistente en la cátedra Lectura y Escritura en Lengua Materna y Lengua Extranjera en la Facultad de Lenguas (Universidad Nacional de Córdoba). Su área de interés es el aprendizaje del vocabulario en el marco de los contextos interaccionales en el ámbito escolar. Ha publicado, en coautoría, libros de actividades para el aula, materiales pedagógicos, capítulos de libros y artículos en revistas especializadas.

Email: alejandramenti@yahoo.com.ar

Florencia Alam es Licenciada en Letras por la Universidad de Buenos Aires. Realizó una maestría en Análisis del Discurso. Actualmente se encuentra finalizando su Doctorado en Educación en la Universidad de Buenos Aires con una beca doctoral del Consejo Nacional de Investigaciones Científicas y Técnicas (CONICET-Argentina)

Para citar este articulo:

Menti, A.B., y Alam, F. (2014). Los gestos y la enseñanza de palabras poco familiares: ¿Cuándo emplean las maestras información gestual? Bellaterra Journal of Teaching \& Learning Language \& Literature, 7(1), 17-32. 